\title{
A Parallel Interval Computation Model for Global Opti- mization with Automatic Load Balancing
}

\author{
Yong $\mathrm{Wu}^{*}$, (吴 勇) and Arun Kumar \\ †Dept. of Int'l Business $\&$ Asian Studies, Griffith University, QLD 4222 Australia \\ ${ }^{\ddagger}$ School of Aerospace, Mechanical $\&$ Manufacturing Engineering, RMIT Uni., Melbourne Australia
}

Email: yong.wu@griffith.edu.au,wuyong@pmail.ntu.edu.sg, a.kumar@rmit.edu.au

\begin{abstract}
In this paper, we propose a decentralized parallel computation model for global optimization using interval analysis. The model is adaptive to any number of processors and the workload is automatically and evenly distributed among all processors by alternative message passing. The problems received by each processor are processed based on their local dominance properties, which avoids unnecessary interval evaluations. Further, the problem is treated as a whole at the beginning of computation so that no initial decomposition scheme is required. Numerical experiments indicate that the model works well and is stable with different number of parallel processors, distributes the load evenly among the processors, and provides an impressive speedup, especially when the problem is time-consuming to solve.
\end{abstract}

Keywords parallel processing, automatic load balancing, computation model, interval analysis, global optimization

\section{Introduction}

In this research, we consider the global optimization problem over the search domain $\mathcal{D} \subset \mathbb{R}^{n}$ which is defined by the lower and upper bounds of all variables:

$$
\begin{array}{cl}
\max & f(\mathbf{x}) \\
\text { s.t. } & g_{i}(\mathbf{x}) \leq 0, i=1, \ldots, r \\
& h_{j}(\mathbf{x})=0, j=r+1, \ldots, s \\
& \mathbf{x} \in \mathcal{D} .
\end{array}
$$

where $f: \mathcal{D} \rightarrow \mathbb{R}$ is the objective function, $g_{i}: \mathcal{D} \rightarrow \mathbb{R}, i=1, \ldots, r$ and $h_{j}: \mathcal{D} \rightarrow \mathbb{R}$, $j=r+1, \ldots, s$ are the inequality and equality constraints, respectively.
Due to the nonlinearity of the objective function and constraints expressed in (1), some effort must be spent if global optimal solutions are wanted.

A large class of global optimization algorithms have adopted divide and conquer (partitioning) approaches. Partitioning approaches divide given domains into smaller sub-spaces whose potential of holding the global optimum is either determined by deterministic approaches, such as [1]; or by stochastic approaches, such as [2].

Deterministic partitioning algorithms can be classified into two major categories. The first category includes algorithms based on interval methods, such as [3] and [4]. These algo- 
rithms use interval arithmetics to calculate the range of the objective function and constraints for a specific partition and guarantee reliable box disposal. The second category includes algorithms based on certain a priori assumptions on functions, such as Lipschitz methods [1, 5], which generate a partition of the domain based on an assumed rate of change, i.e., the Lipschitz constant. The partition disposal can be reliable if the Lipschitz constant is known with accuracy.

Stochastic partitioning methods assess each partition with certain criteria and subdivide those partitions which are more "promising." Sub-spaces can be selected based on possible largest expected increase in function values (e.g. [6]); or based on fuzzy assessment (e.g. [2]) where evidence within each partition is collected by using random search techniques.

Interval methods [3, 7], as a deterministic partitioning approach, have the advantage of providing explicit ranges over functions to guarantee assured interval disposal. An interval hypercube, which is commonly referred as 'box') can be discarded or removed from consideration when it is verified that there are no better solutions inside it or it is infeasible, or the objective function range over the hypercube is less than some small value, $\varepsilon_{f}[7]$.

Branch-and-bound technique is usually employed for interval methods. It should be noted that in branch-and-bound the number of boxes to be managed usually increases exponentially during execution. This requires both efficient box disposal procedures and powerful box management schemes and computation models. A good candidate for speeding up the computation is parallel processing since each branch can be assessed separately. Some parallel models divide the root problem into a certain number of subproblems before execution to enable each processor has one problem to process. However, due to the fact that no knowledge of the execution behavior is known a pri- ori, it is not suitable to statically balance the load at the very beginning. Furthermore, it is difficult to achieve an optimal initial decomposition [8] even when it is practical. Therefore, parallel computation models must address the load balancing problem.

In this study, we propose a decentralized parallel interval computation model (PICM) for global optimization. The model eliminates the requirement for an initial decomposition scheme to feed every processor at the beginning. It also automatically balances the workload among processors through alternative message passing. These two characteristics make the parallel model easy to implement.

The remainder of the paper is organized as follows: Section 2 briefly introduces interval analysis, constraint handling and some related parallelization approaches; Section 3 presents the PICM model and analyzes the model properties; Section 4 conducts numerical experiments to show the performance of PICM and Section 5 concludes this paper.

\section{Interval Analysis and its Paralleliza- tion}

Interval analysis provides function ranges which cover the objective function and constraints ranges over a hypercube (box) and can be easily embedded into a branch-and-bound computation model. The whole search area can be consecutively subdivided into smaller search areas - branching. Whereas the interval arithmetic provides the function ranges - bounding, which eliminates certain areas that surely do not hold the global optima. A prototype of branch-and-bound for global optimization using interval analysis is presented in Algorith$\mathrm{m}$ 1. The whole search space is first treated as a single interval (box), which is subsequently divided into several sub-intervals. The procedure is then recursively applied to those subintervals and results in a tree of search nodes 


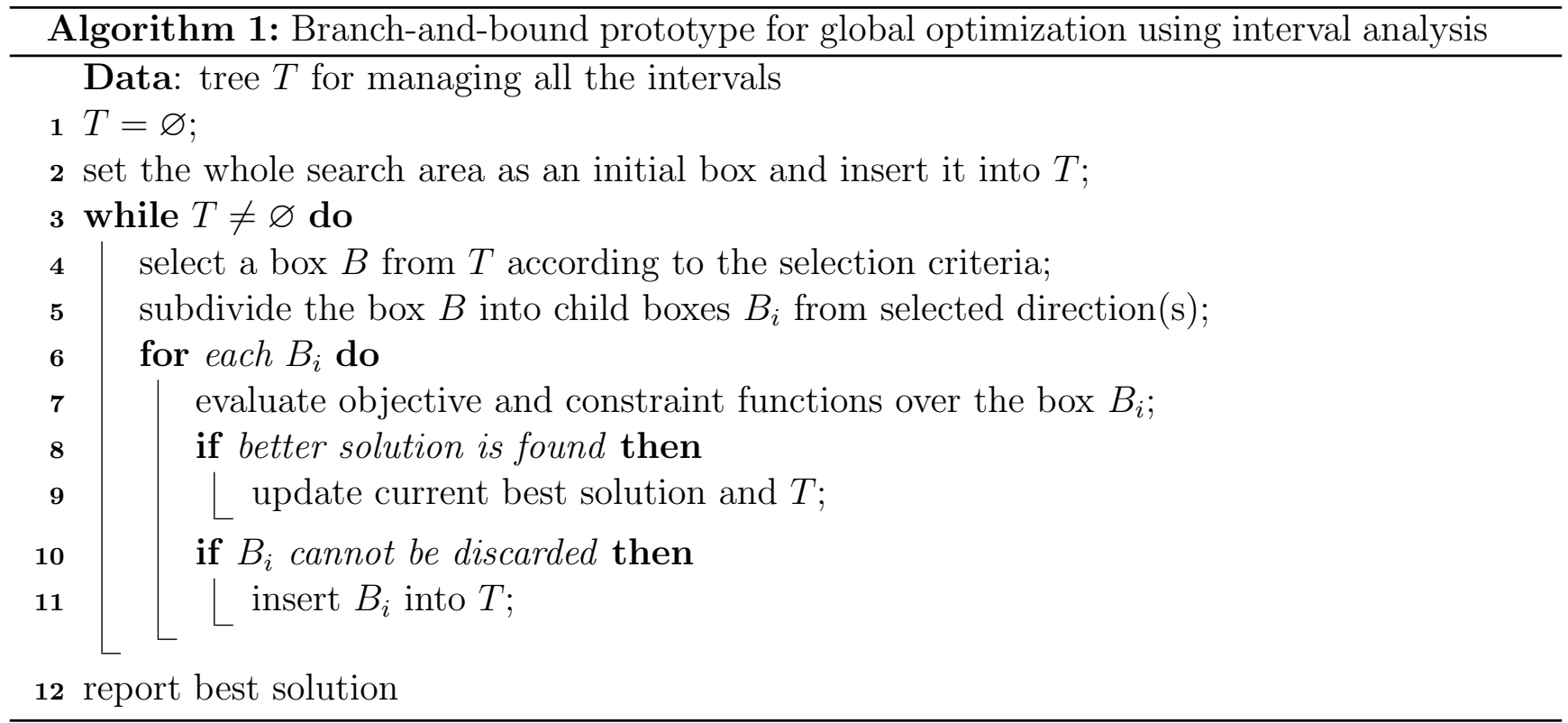

(sub-intervals).

Many strategies have been investigated for Steps 4 and 5 in Algorithm 1, which correspond to the two problems that need to be considered when implementing the branch-and-bound prototype: the first is which branch should be selected for processing (branch selection criteria) and the second is how a branch should be partitioned (branching rules). There are some strategies proposed for branch selection: some have long been used in interval methods, such as the strategy to select the box with the best upper bound [9], select the box which is the oldest [10], and select the box which has maximum width [10] and so on. Once the box is selected, it will be partitioned along one or several of its edges [3], such as subdividing along the widest edge [11]. There are other rules that utilize the gradient, such as the rule of Hansen and Walster [3] and the rule of Ratz [12]. Some heuristic selection strategies have also been proposed in $[13,14,15]$. Those strategies have substantially improved the performance of interval analysis. Recent implementations of interval methods can be found in, for example, [16] and [17]. In the rest of this paper, we will not focus on the selection and subdivision strategies since the main objective of this paper is the parallelization of interval analysis.

In the presence of constraints, the feasibility of a box is checked by examining the range of each constraint. It should be noted that when dealing with equality constraints, it is almost computationally impossible to satisfy them [7]. In practical computation, the equality constraints can be relaxed and replaced by:

$$
-\varepsilon \leqslant h_{i}(x) \leqslant \varepsilon \quad i=r+1, \ldots, s,
$$

for some positive small valued $\varepsilon$. Similarly, the inequality constraints can also be relaxed:

$$
g_{i}(x) \leqslant \varepsilon \quad i=1, \ldots, r .
$$

Thus, infeasible boxes can be immediately discarded, and feasible boxes can be kept for later processing if their upper bounds are superior to the known best solution. Since the constraints are also evaluated by interval arithmetic, there exist certain boxes which are neither totally feasible nor completely infeasible. Those boxes are called "pending boxes."

Efforts for parallelization of interval computation include centralized and decentralized models. Master/slave architecture (centralized 
model), such as the one in [18], has the advantage of efficiently utilizing available information. However, the master processor may easily become a bottleneck for the whole computation model due to data management and communication overheads, especially when the number of processors is large. Decentralized models, on the other hand, do not have such disadvantages. However, they need effective ways to exchange workload so that the total processor idle time can be minimized. Different load balancing mechanisms have been proposed. Such as, Eriksson and Lindstrom [19] investigated dynamic load balance schedulers. Benyoub and Daoudi [20] proposed an approach based on synchronization and uniform redistribution of boxes. Ibraev [21] presented a challenge leadership model where each processor has the chance to become the "leader" when it finds better solution. Gau and Stadtherr [22] provided a stage-wise asynchronous workload information exchange strategy for dynamic load balancing. Berner [23] presented a parallel method using a centralized mediator for the dynamic load balancing. A common observation from these approaches is that the load balancing makes the management of communication a complex task and subsequently complicates the parallel model implementation.

\section{The Parallel Model}

\subsection{Model Topology}

Suppose there are $p$ processors in total and each processor is assigned a rank ranging from 0 to $p-1$. The alternative message passing mechanism proposed in this paper sends messages to and receives messages from processors which are a hop away. The hop, $h$, changes in a periodic way according to a series of $2^{0}, 2^{1}, 2^{2}, \cdots, 2^{C-1}$, where $C=\operatorname{round}\left(\log _{2} p\right)$. The round operation helps the cases where the number of processors does not equal to an integral power of 2. Thus, one particular processor sends messages to and receives messages from a group of processors alternatively and therefore the mechanism is referred as alternative message passing.

The rank of the processor to which processor $P_{i}$ should send message can be represented as $(i+h) \bmod p$, and the rank of the processor from which a message should be received can be denoted as $(i-h+p) \bmod p$. Taking a parallel environment with 8 processors as an example, the alternative message passing can be represented in Fig. 1. The operations with $h=1,2,4$ constitute a complete cycle of alternative message passing since $C=3$ when there are 8 processors.

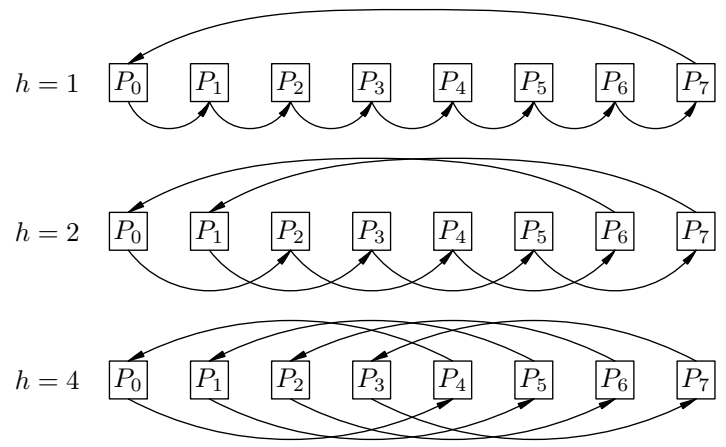

Fig. 1. The alternative message passing.

\subsection{Communications, Message Format and Processing}

MPI [24] is adopted for message passing in this work. There are two method$\mathrm{s}$ for communicating data in MPI: blocking and non-blocking. In blocking communication, the sending process does not return until the send buffer is available for reuse. In nonblocking communication, the sending process returns immediately, and may only have started the message transfer operation, not necessarily completed it. The application may not safely reuse the message buffer after a nonblocking routine returns. However, the advantage of non-blocking routines is that they enable the communication overlap with the com- 
putation [24]. In this research, both blocking and non-blocking message passing operations are employed.

\begin{tabular}{|l|l|l|l|}
\hline box & $\tilde{f}^{*}$ & status array & tag \\
\hline
\end{tabular}

Fig. 2. The message format used by PICM.

The content of the messages passed among processors is as shown in Fig. 2. Each message includes a box, the current best local solution, $\tilde{f}^{*}$ on the local processor, a status array which records all the processors' status, and a message tag for destination processor to choose corresponding message handling operations. The content of the 'box' section depends on the current status of the message sending processor. If the binary tree $T$, which stores all the boxes to be processed, is not empty, a box is selected according to the box selection rules; otherwise, the 'box' section will be marked as empty but the message will still be sent out. Sending a message with empty 'box' section keeps the message flow continuous and the order of alternative message passing consistent with other processors. Correspondingly, different message tags are used to instruct the receiving processor to choose the proper message handling operations. There are three tags used: the message tag BOx indicates a box has been sent/received; the message tag IDLE means the message contains no box; and the message tag QUIT instructs the receiving processor to quit the computation.

Sending the current best local solution $\tilde{f}^{*}$ and the processor status array with each message avoids the time-consuming broadcasting operations. The processor status array is used for termination detection since non-blocking message passing makes the termination detection a tricky task. The status array is set by each processor according to its current status. Algorithm 2 describes the operations for message sending. The best known solution on the current processor is first packed into the message $M_{s}$, followed by the box information. If the binary tree $T$ is not empty, the tag is set to BOx and a box $B$ is retrieved from $T$ for sending. At the same time, the status of the current processor and its destination processor are set to non-idle in the status array; otherwise, the send tag is set to IDLE and the status of current processor is set to idle in the status array.
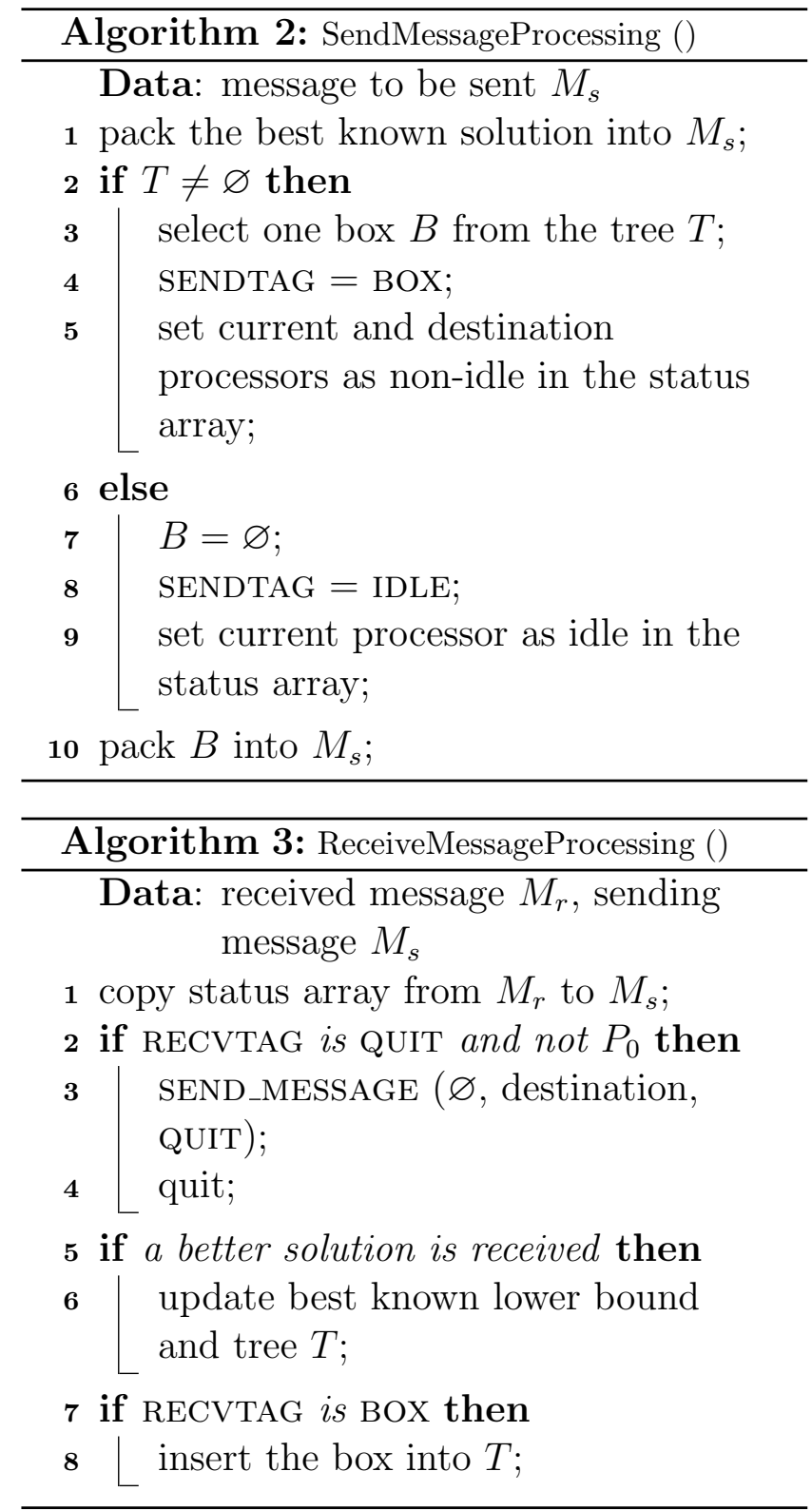

Algorithm 3 describes the operations for processing received messages. The status array is first copied from received message $M_{r}$ to the status array of the next sending message $M_{s}$. 
The messages are then handled by examining the message tag (RECVTAG). If the tag is QUIT, the processor sends a QUIT signal to its destination processor and exits. If a better solution is received, the best known solution on this processor and the binary tree $T$ are updated. If the RECVTAG is BOX, the box is inserted into the tree $T$ for processing.

\subsection{The Parallel Algorithm}

In PICM, each processor maintains a binary tree $T$ to manage the boxes. Initially, PICM sets the whole search space as a single problem and stores it at processor $P_{0}$, the tree $T$ on other processors are empty at the beginning.

The the parallel model PICM is shown in Algorithm 4. We define computation cycle as the cycle from one operation to the next same operation for ease of description (Step 6 to 29). The message sending flag $F_{s}$ and message receiving flag $F_{r}$ are used to check the status of non-blocking message passing operations. The status can either be SENT and WAITING for $F_{s}$ and RECEIVED and WAIting for $F_{r}$. Small capital letters are used for description of message passing operations in a simplified way, and "NB" indicates them are non-blocking message passing ones. The simplified operations need to specify content, origin/destination, message tag, and message status flag.

All processors start with a non-blocking message receiving operation except the processor $P_{0}$, which first sends a message out, then starts the non-blocking message receiving operation. This initiates the message flow among all processors. In each computation cycle, first the message receiving flag $F_{r}$ is checked. If a message has arrived, the message is processed according to Algorithm 3 and another nonblocking receiving operation is started subsequently. Otherwise, if the binary tree $T$ is empty, the processor waits until a message is received and processes the message according to Algorithm 3 and starts another non-blocking receiving operation. In the processing phase, if the binary tree $T$ is not empty, a box is selected and partitioned; the child boxes are subsequently evaluated and either inserted back into $T$ if they are pending or feasible, or discarded when they are infeasible or inferior to the best known solution. Of course, if a better solution is found during box splitting, the current best solution and binary tree $T$ need to be updated. Subsequently, the processor prepares a message according to Algorithm 2 and sends the message out if a message has been received in this computation cycle. The message sending flag $F_{s}$ is then checked, in case the binary tree $T$ is empty and $F_{s}$ is WAITING, the processor waits until this message sending operation finishes. By performing non-blocking sending and receiving, the communication time in each computation cycle can be shortened. When the non-blocking message passing operations in previous computation cycles are not finished, the current computation cycle will only consists of interval processing. Thus, each computation cycle may have message passing operations or not, depending on the status of previous message passing operations.

The termination detection is done by processor $P_{0}$. It should be noted that the status change of a particular processor is not reflected among all processors at the same time. Instead, it is passed through message passing which in turn prevents wrong termination detection. When the status array of all processors in $P_{0}$ 's sending status array are idle, $P_{0}$ sends a QUIT signal out. Consequently, the QUIT signal is passed across all processors to terminate them. The use of non-blocking message passing makes the termination detection complex since even when all processors are currently idle, it is not sufficient to terminate the computation due to the messages in transit. These messages in transition will reactivate idle processors. 


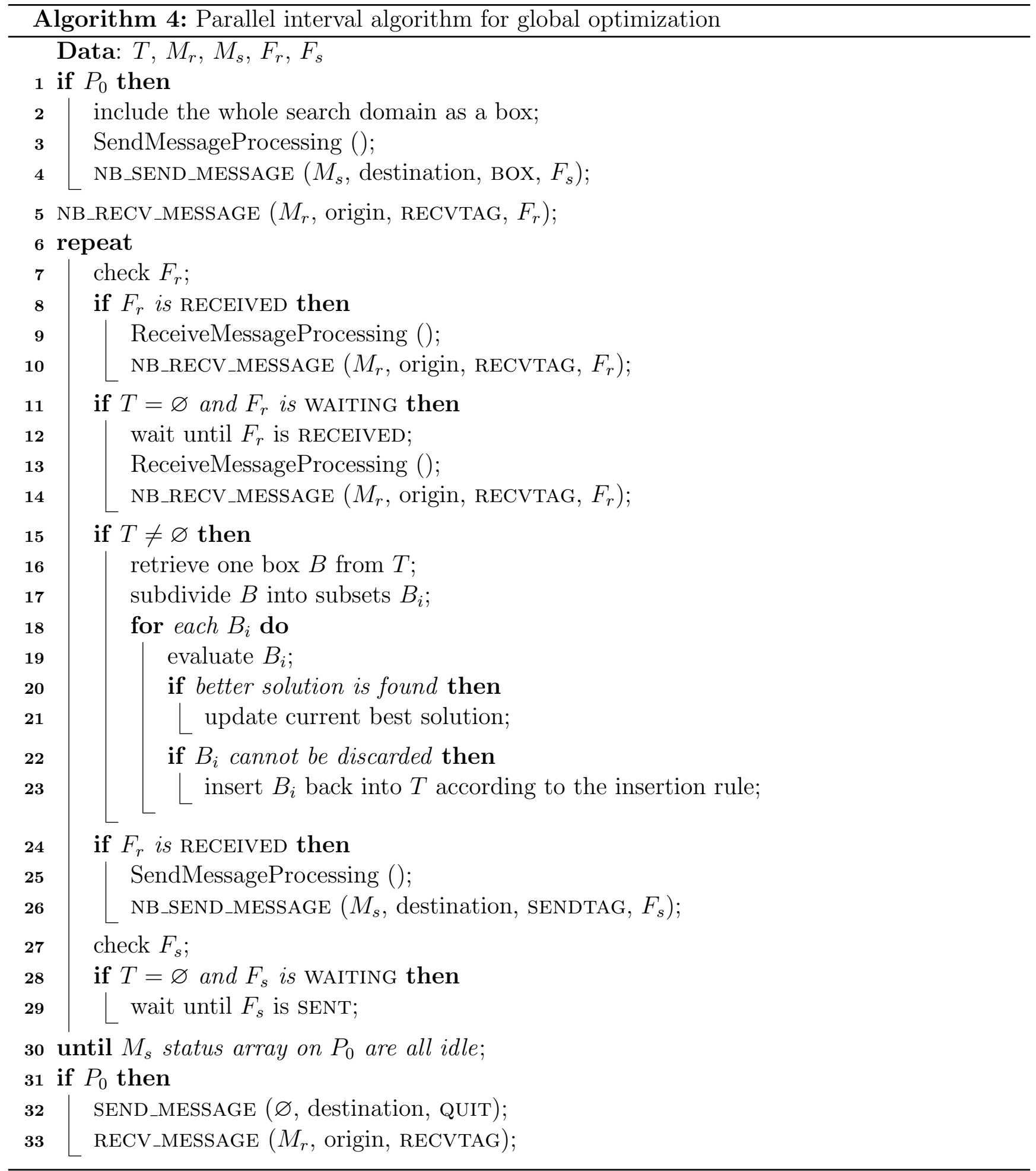

\subsection{Model Properties}

In PICM, although the alternative message passing operations look simple, the mod- el itself does exhibit some powerful properties. We define the concept of dominance for a box as follows: 
Definition 1. A local dominant box is a box which should be selected according to the selection rules among all boxes on the processor it locates.

Definition 2. A global dominant box is a box which should be selected according to the selection rules among all boxes on all processors.

In serial computation, a local dominant box, which at the same time is also a global dominant one, is selected for next subdivision according to the given subproblem selection rules. In PICM, each processor first send$\mathrm{s}$ its local dominant box to another processor and processes the local dominant node after the message received has been handled. This makes the dissemination of the globally dominant boxes easily achievable and ensures high qualify (dominant) boxes are processed with priority.

The properties of PICM utilizing $p$ processors can be summarized as follows:

Property 1: If a box is globally dominan$\mathrm{t}$ and its child boxes are locally dominant on each corresponding processor, then child boxes split from this globally dominant box will dominate all processors after round $\left(\log _{2} p\right)$ times of message passing provided no child box will be discarded during the computation.

Bear in mind that PICM sends a box out before processing intervals in a computation cycle, and the alternative message passing enables/forces a processor to send messages to different processors within any given round $\left(\log _{2} p\right)$ times of message passing, Property 1 is actually equivalent to the calculation of the depth of a balanced binary tree at the level where at most $p$ nodes can exist.

Without loss of generality, suppose currently the processor $P_{0}$ has such a globally dominant node, $N_{g}$, and the hop $h=1$. Since $N_{g}$ is globally dominant on $P_{0}$, it is selected for subdivision and one child node of $N_{g}$ is sent to $P_{1}$ since it is locally dominant. That is, after 1st message passing, processors $P_{0}$ and $P_{1}$ have nodes split from $N_{g}$. For the second message passing, the hop now becomes $h=2, P_{2}$ and $P_{3}$ will get such nodes from $P_{0}$ and $P_{1}$, respectively. It can be easily verified that the number of processors having such nodes will grow exponentially and without repetition. Eventually, after round $\left(\log _{2} p\right)$ message passings, all processors will acquire a node split from $N_{g}$ once the local dominant property of the child nodes holds and no child box will be discarded during the computation.

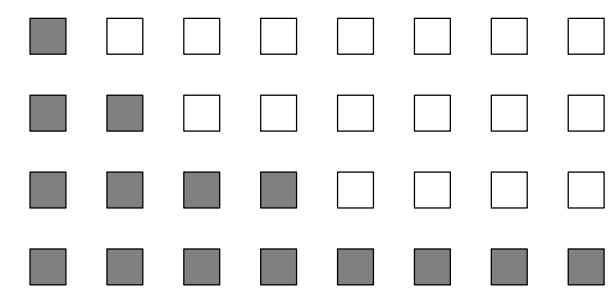

Fig. 3. The pervade of nodes among all processors

Property 1 shows that the children of a globally dominant node will be processed by all processors if they cannot be discarded and locally dominant in round $\left(\log _{2} p\right)$ times of message passing. Fig. 3 illustrates such a case with 8 processors. It can be easily verified with the help of Fig. 1. This property ensures that more promising nodes are processed as early as possible among all processors. This power is especially important when the number of processors is large. For example, it only takes 10 message passings to disseminate the children of a node in Property 1 in a parallel system with 1024 processors.

Property 2: A processor can only be idle after at most round $\left(\log _{2} p\right)$ times of message passing consecutively if there is at least one node left among all processors and at least one of the child nodes split from that node cannot be discarded.

Similar to the proof for Property 1, the proof for Property 2 is equivalent to the problem of calculating the maximum possible depth 
for a number $i$ (where $0 \leqslant i \leqslant p$ and represents the rank for processor $P_{i}$ ) first appearing in a balanced binary tree where each level is full of nodes and numbered consecutively.

Property 2 indicates that the maximum consecutive idle time a processor might have when there is a node that has Property 1 among all processors. Actually, when there is only one node left, that node is for sure globally dominant and the child nodes will pervade among all processors.

Property 3: If all processors are not idle, computation cycles on each processor will cost almost the same amount of time and the same data managing complexity.

In each computation cycle, the time spen$\mathrm{t}$ on sending-receiving phase of each processor is the same due to the non-blocking sending and receiving operations. The computation phase costs almost the same amount of time since each processor manipulates a homogeneous problem due to the characteristics of branch-and-bound algorithms.

Property 3 indicates that when the workload is evenly distributed among all processors, the time needed by each processor will be almost identical. Thus, when total effort needed is not increased, the processing time can be reduced correspondingly since each processor will only need to process a portion of the total effort required by a serial implementation and achieve a good performance on parallel efficiency.

\section{Numerical Experiments}

\subsection{Test problems}

Sixteen constrained global optimization problems are selected from the literature to evaluate the performance of PICM. Table 1 summarizes the dimensions, type and number of constraints (where $E$ means equality constraints and $I$ means inequality constraints), and the references of the test problems. Detail description of the test problems is available upon request.

As we mentioned earlier in the paper, the main purpose of this paper is to evaluate the performance of the parallel computation model PICM; therefore we apply rather simple interval strategies from the interval computational perspective. The box with best upper bound is used as the box selection criteria and bisection is applied for box subdivision. The subdivision direction is selected according to the infeasibility index presented in [25]. Some accelerating devices are introduced to speedup the convergence of interval methods, such as the midpoint test which evaluates the middle point of a box. The monotonicity test (see e.g. [3] or [26]) is not used since it is not applicable to the test problems.

Table 1. Summary of test problems.

\begin{tabular}{llll}
\hline Prob. & Dim. & Cons. & Reference \\
\hline 1 & 5 & $3 E$ & Sect. 8.2.7 [27] \\
2 & 6 & $4 E, 1 I$ & Sect. 8.2.8 [27] \\
3 & 5 & $6 I$ & Sect. 3.3 [27] \\
4 & 2 & $3 I$ & Sect. 12.2.6 [27] \\
5 & 2 & $3 I$ & Revised from Spike [28] \\
6 & 5 & $2 E, 3 I$ & Sect. 12.2.1 [27] \\
7 & 3 & $3 I$ & Sect. 12.2.2 [27] \\
8 & 5 & $6 I$ & Sect. 7.2.5 [27] \\
9 & 4 & $1 E, 1 I$ & Revised from Rastrigin $[29]$ \\
10 & 2 & $3 I$ & Based on Crescent [30] \\
11 & 8 & $6 I$ & Sect. 5.4.2 [27] \\
12 & 6 & $4 E, 1 I$ & Sect. 7.2.2 [27] \\
13 & 6 & $6 I$ & Sect. 3.4 [27] \\
14 & 8 & $1 E, 1 I$ & Revised from Rastrigin [29] \\
15 & 10 & $1 E, 1 I$ & Revised from Rastrigin [29] \\
16 & 20 & $14 E$ & CUTEr [31] \\
\hline
\end{tabular}

The parallel computation model is tested in a cluster of up to 16 processors $\left(\right.$ Intel $^{\mathrm{R}}$ $\mathrm{Xeon}^{\mathrm{TM}} 3.06 \mathrm{GHz}$ ) running on Red Hat Linux 9.0. The MPI version used in the numeri- 
cal experiments is MPICH-G2 and the interval package used is PROFIL/BIAS [32].

\subsection{Computation Results}

Table 2 presents the numbers of objective and constraint function evaluations and the maximum binary tree sizes for each problem using 1, 2, 4, 8 and 16 processors, respectively. Eight problems are selected to further assess the performance of PICM when there are more processors presented (via simulating the runs with 32 and 64 processors by running 2 and 4 processes on a single processor, respectively). Further, we randomly choose two prime numbers 13 and 37, to evaluate PICM's performance with arbitrary number of processors when the number of processors is not integral power of 2 . These results are italicized in Table 2 .

It can be observed that in most cases PICM uses about the same amount of function and constraint evaluations (with slight fluctuations) for parallel computations compared with the serial one, sometimes even less. However, some problems utilize different numbers of objective and constraint evaluations, such as Problems 7, 11 and 16. The number of interval evaluations changes drastically in some occasions. The reason to this phenomenon might be that PICM processes the boxes in a different order. In serial implementation, only the box with global dominant property can be processed in each iteration. While in parallel implementation, the second-best box at a particular processor has the chance to be processed since the best box might have been packed to be sent out. Such effects are easier to be observed from higher dimensional problems. Overall, Table 2 indicates that PICM does not increase the effort needed to solve the selected test problems. From the results we can conclude that although the problem is treated as a single one at the beginning, the alternative message passing mechanism makes it possible to rapidly dis- tribute subproblems and the exchange of local dominant nodes effectively controls the total effort required by PICM.

The maximum binary tree size, on the other hand, demonstrates the effect of automatic load balancing of PICM. As more and more processors participate the computation, it would be reasonable to assume that the maximum binary tree size will decrease. The data in Table 2 confirms this assumption. It can be observed that the maximum binary tree sizes change almost inversely proportional to the number of parallel processors, especially for those problems which need big binary trees. There is only one exception, Problem 11, where the binary tree size does not change very much but remain close to the serial case. Compare with the interval evaluations required for Problem 11, it can be concluded that the search space of Problem 11 is explored quite differently when different number of processors are used. It is worthwhile to point out that the maximum binary tree sizes for Problems 7, 14, 15 and 16 are more than halved when the number of processors are doubled in some occasions. Overall, the data indicates that PICM distributes the workload quite evenly among all processors.

The italicized data for selected problems for $13,32,37$ and 64 processors present similar pattern as we observed above: the numbers of function and constraint evaluations remain stable for the newly tested number of 'processors'. The selection of prime numbers of processors does not exhibit an impact on the performance and the trend on the maximum binary tree size remains the same, i.e., as more processors are used, the tree size decreases.

Fig. 4 presents the CPU time (in second$\mathrm{s}$, shown on top of the three sub-figures) used for the serial implementation and the parallel efficiency (not speedup) for all test problems. In general, the test problems can be classified into three groups. The first group includes Problems 3, 4, 5, 6, 7 and 10, where sub-linear 
Table 2. Objective function and constraint evaluations, maximum binary tree size

\begin{tabular}{|c|c|c|c|c|c|c|c|c|c|}
\hline Problem & 1 & 2 & 4 & 8 & 13 & 16 & 32 & 37 & 64 \\
\hline \multicolumn{10}{|c|}{ Objective function evaluation } \\
\hline$\overline{1}$ & 169554 & 169558 & 169179 & 169618 & 168815 & 169845 & 170015 & 168945 & 168469 \\
\hline 2 & 181823 & 181886 & 177336 & 178013 & 181902 & 177318 & 173767 & 169488 & 182695 \\
\hline 3 & 25293 & 25235 & 25101 & 25240 & 25244 & 25104 & 25569 & 25405 & 26110 \\
\hline 4 & 146 & 154 & 177 & 155 & - & 155 & - & - & - \\
\hline 5 & 263 & 273 & 273 & 273 & - & 267 & - & - & - \\
\hline 6 & 16229 & 16224 & 16062 & 16207 & 16314 & 16279 & 15106 & 15008 & 16650 \\
\hline 7 & 3120 & 4685 & 3874 & 3670 & - & 2520 & - & - & - \\
\hline 8 & 74239 & 74152 & 74293 & 74512 & 74742 & 73833 & 73262 & 73977 & 75105 \\
\hline 9 & 54138 & 54116 & 53636 & 51534 & 52343 & 52742 & 49496 & 48804 & 47192 \\
\hline 10 & 9878 & 9877 & 9790 & 9742 & 9716 & 9733 & 9657 & 9758 & 9833 \\
\hline 11 & 693668 & 497564 & 192572 & 130421 & - & 219501 & - & - & - \\
\hline 12 & 365081 & 361237 & 322973 & 326116 & - & 357013 & - & - & - \\
\hline 13 & 146151 & 146152 & 146753 & 149256 & 150168 & 148859 & 152315 & 151243 & 156491 \\
\hline 14 & 309684 & 358220 & 335139 & 282347 & - & 286554 & - & - & - \\
\hline 15 & 1866677 & 2144400 & 1798656 & 1766403 & - & 1610199 & - & - & - \\
\hline 16 & 1181529 & 1850162 & 2120874 & 562662 & - & 879626 & - & - & - \\
\hline \multicolumn{10}{|c|}{ Constraint evaluation } \\
\hline 1 & 815690 & 815688 & 813957 & 815869 & 812212 & 816909 & 817718 & 812880 & 810821 \\
\hline 2 & 1394046 & 1393715 & 1363809 & 1368333 & 1394413 & 1363054 & 1339103 & 1311473 & 1399550 \\
\hline 3 & 88455 & 88257 & 88011 & 88320 & 88408 & 87965 & 89368 & 88810 & 90914 \\
\hline 4 & 566 & 580 & 644 & 583 & - & 583 & - & - & - \\
\hline 5 & 365 & 371 & 371 & 371 & - & 371 & - & - & - \\
\hline 6 & 60503 & 60510 & 60098 & 60532 & 60682 & 60750 & 56901 & 56790 & 62308 \\
\hline 7 & 51 & 369 & 648 & 408 & - & 366 & - & - & - \\
\hline 8 & 274353 & 274121 & 274907 & 275214 & 276358 & 273372 & 271730 & 273712 & 277331 \\
\hline 9 & 96483 & 96536 & 95632 & 91768 & 93445 & 94136 & 88792 & 87709 & 85385 \\
\hline 10 & 216 & 222 & 221 & 233 & 237 & 233 & 233 & 237 & 237 \\
\hline 11 & 31805 & 118169 & 138600 & 249322 & - & 304920 & - & - & - \\
\hline 12 & 2765102 & 2738602 & 2484645 & 2504490 & - & 2720016 & - & - & - \\
\hline 13 & 542725 & 542795 & 544773 & 552040 & 554779 & 550459 & 560510 & 554896 & 562788 \\
\hline 14 & 507397 & 586906 & 551975 & 459382 & - & 467326 & - & - & - \\
\hline 15 & 3044586 & 3500170 & 2977285 & 2904029 & - & 2649758 & - & - & - \\
\hline 16 & 23662 & 22048079 & 18325953 & 4096324 & - & 733156 & - & - & - \\
\hline \multicolumn{10}{|c|}{ Maximum binary tree size } \\
\hline 1 & 13244 & 6631 & 3612 & 1858 & 1257 & 1068 & 516 & 442 & 287 \\
\hline 2 & 11486 & 5934 & 3302 & 1689 & 1073 & 860 & 487 & 410 & 261 \\
\hline 3 & 3489 & 1768 & 897 & 487 & 317 & 232 & 144 & 120 & 87 \\
\hline 4 & 9 & 6 & 4 & 3 & - & 3 & - & - & - \\
\hline 5 & 26 & 14 & 10 & 4 & - & 3 & - & - & - \\
\hline 6 & 3964 & 2001 & 1005 & 526 & 320 & 273 & 136 & 126 & 81 \\
\hline 7 & 850 & 344 & 225 & 80 & - & 39 & - & - & - \\
\hline 8 & 10378 & 5656 & 2855 & 1405 & 978 & 735 & 391 & 359 & 220 \\
\hline 9 & 7941 & 3997 & 2077 & 1060 & 657 & 581 & 294 & 276 & 158 \\
\hline 10 & 505 & 268 & 155 & 92 & 63 & 50 & 27 & 28 & 17 \\
\hline 11 & 2,191 & 1,942 & 2,131 & 2,878 & - & 2,033 & - & - & - \\
\hline 12 & 16,146 & 9,363 & 4,125 & 3,912 & - & 3,816 & - & - & - \\
\hline 13 & 30939 & 15530 & 8171 & 4301 & 2651 & 2233 & 1199 & 1074 & 660 \\
\hline 14 & 87,519 & 43,380 & 20,095 & 4,783 & - & 3,499 & - & - & - \\
\hline 15 & 544,816 & 273,087 & 91,584 & 41,134 & - & 13,588 & - & - & - \\
\hline 16 & 20,771 & 15,482 & 12,485 & 7,531 & - & 802 & 一 & - & - \\
\hline
\end{tabular}




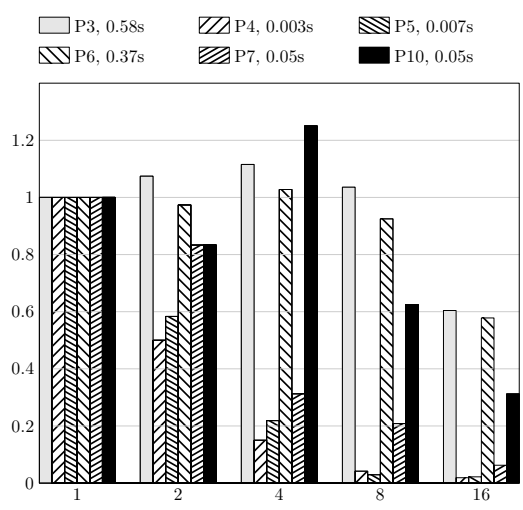

(a) Sub-linear speedup cases

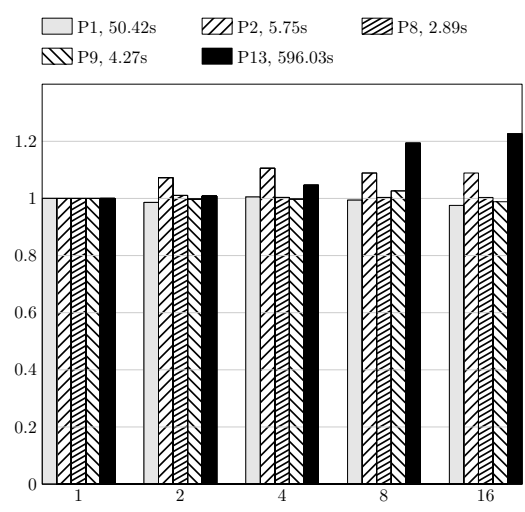

(b) Linear speedup cases

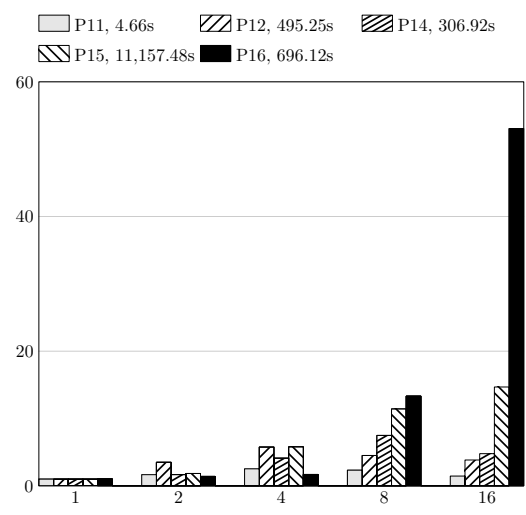

(c) Super-linear speedup cases

Fig. 4. Parallel efficiency of PICM

speedup (parallel efficiency $<1.0$ ) is observed, i.e., the speedup deteriorates as the number of processors increases. However, we should point out that for each problem in this group, the time required for the serial implementation is less than 1 second. Of course, in practical computation, there is no need (or not worth) to apply parallel computation to such problems. However, the results are presented here to demonstrate that the performance of PICM is not significantly affected considering the overhead of parallel initialization and message passing. The second group includes Problems 1, 2, 8,9 and 13, where a linear speedup (efficiency $=1.0$ ) can be observed. The last group includes Problems 11, 12, 14, 15 and 16, where super linear speedup (efficiency $>1.0$ ) can be observed which indicates PICM takes advantage of the parallel implementation and significantly reduces the total effort to solve these problems.

\subsection{Rough comparison with other par- allel models}

It is rather difficult to compare the performance of parallel methods due to the $\mathrm{d}$ ifferences in software packages, interval techniques, computer architectures, test problems, programming languages and sometimes lack of data reported. However, the parallel efficien- cy could be one possible way (since it measures the speedup and scalability) to conduct a rough performance comparison between our approach and other methods from the literature. The performance of these models are briefly listed below:

PICM: Tested with up to 16 processors, simulated with up to 64 processors. Maximum parallel efficiency with 16 processors is 53.1. For problems with serial running time over 110 seconds, the model scales up quite well.

Ibraev [21]: Tested with up to 16 processors, maximum efficiency with 16 processors reached 9.1. Scales up well when serial running time is over $50-100$ seconds.

Eriksson and Lindstrom [19]: Tested with up to 64 processors. For the test results presented in the paper (only three), one exhibits super-linear speedup while the performance for the other two deteriorate quickly when more than 16 processors are employed.

Gau and Stadtherr [22]: Tested with up to 32 processors, maximum efficiency with 16 processors (reproduced from the paper) is about 3.1.

Berner [23]: Tested with up to 32 processors, linear or slightly super-linear speedup for most of the test problems achieved. For problems with serial running time of less than 100 seconds, it was difficult to observe super-linear 
speedup.

The above rough comparison indicates that PICM has a high parallel efficiency and is able to provide decent computational performance. The simulated runs with up to 64 processors also illustrates the scalability of the model. When the number of processors gets larger, it might take some time for all processors in the model to be fully loaded. To get rid of this drawback, the processor $P_{0}$ can conduct a multi-section split (so more boxes are generated at the beginning) and then send out the boxes to some 'far away' processors to speed up the initial load balancing process.

\subsection{Discussion}

The computational performance of PICM might be contributed by several reasons. First, the workload in PICM could be welldistributed among all processors. The maximum tree sizes shown in Table 2 implicitly suggest this. Smaller tree size not only indicates that each processor handles less boxes, but also suggests less data management complexity since the operations of insertion and deletion of boxes will be quicker. This property is especially useful when the number of boxes to be managed is large. Moreover, the requirement for memory is also reduced, which is meaningful for processors with limited memory and extends the model's applicability to more difficult problems. Second, each processor sends a local dominant box to its destination processor and handles its local dominant box after considering the received box from its origin processor. This mechanism helps the model process highquality boxes among all processors. Further, sending local dominant boxes also helps update the best solution in each processor without the time-consuming broadcasting operation. Finally, as discussed in Section 4.2, PICM might be able to explore the search space in a different and more efficient way from that of serial implementation. This effect is more conspicuous when the problem dimension is high since it normally takes longer (more message passing between processors) to split a high dimensional box into disposable small ones.

\section{Conclusion}

In this research, a decentralized parallel model for global optimization using interval analysis is presented. The model is adaptive to any number of processors and there is no need to consider initial decomposition of the problem. By performing the alternative message passing with different hop steps, the workload is rapidly and evenly distributed among al1 processors participating in the computation. Moreover, the communication operations are easy to handle which simplifies the implementation of the parallel model. Numerical experiments indicate that PICM not only speeds up the computation, but also explores the search space in an efficient way, the performance is especially noticeable when the problems are difficult and time-consuming to solve. The model proposed in this paper should have no difficulty to be applied to any other kind of branch-andbound problems.

\section{Acknowledgement}

We thank the two anonymous reviewers for their constructive and pertinent comments, which have greatly improved the content and the presentation of this paper.

\section{References}

[1] Hansen P, Jaumard B. Lipschitz optimization. In Handbook of Global Optimization, Horst R, Pardalos P M (eds.), Dordrecht: Kluwer Academic Pulishers, 1995, pp.407493.

[2] Özdamar L, Demirhan M B. Experiments with new stochastic global optimization 
search techniques. Computers \& Operations Research, 2000, 27:841-865.

[3] Hansen E. Global Optimization Using Interval Analysis. New York: Marcel Dekker, 1992.

[4] Kearfott R B. Rigorous Global Search: Continuous Problems. Dordrecht: Kluwer, 1996.

[5] Pintér J. Branch and bound algorithms for solving global optimization problems with lipschitzian structure. Optimization, 1988, 19:101-110.

[6] Tang Z B. Adaptive partitioned random search to global optimization. IEEE Transactions on Automatic Control, 1994, 39(11):2235-2244.

[7] Ratschek H, Rokne J. Interval methods. In Handbook of Global Optimization, Horst R, Pardalos P M (eds.), Dordrecht / Boston / London: Kluwer Academic Publishers, 1995, pp.751-828.

[8] Skillicorn D B, Talia D. Models and languages for parallel computation. ACM Computing Surveys, 1998, 30(2):123-169.

[9] Skelboe S. Computation of rational interval functions. BIT, 1974, 14:87-95.

[10] Hansen E, Sengupta S. Global constrained optimization using interval analysis. In Interval Mathematics, Nickel K (ed.), Berlin: Springer-Verlag, 1980, pp.25-47.

[11] Moore R E. Interval Analysis. Engelwood Cliffs: Prentice Hall, 1966.

[12] Ratz D, Csendes T. On the selection of subdivision directions in interval branchand-bound methods for global optimization. Journal of Global Optimization, 1995, 7:183-207.
[13] Casado L G, García I, Csendes T. A heuristic rejection criterion in interval global optimization algorithms. BIT $\mathrm{Nu}$ merical Mathematics, 2001, 41(4):683692.

[14] Casado L G, Martínez J A, García I. Experiments with a new selection criterion in a fast interval optimization algorithm. Journal of Global Optimization, 2001, 19:247-264.

[15] Csendes T. New subinterval selection criteria for interval global optimization. Journal of Global Optimization, 2001, 19:307-327.

[16] Pedamallu C S, Özdamar L, Csendes T, Vinkó T. Efficient interval partitioning for constrained global optimization. Journal of Global Optimization, 2008, 42(3):369384 .

[17] Sun M. A fast memoryless interval-based algorithm for global optimization. Journal of Global Optimization, 2010, 47(2):247271.

[18] Henriksen T, Madsen K. Use of a depthfirst strategy in parallel global optimization. Technical Report 92-10, Institute of Numerical Analysis, Technical University of Denmark, Lyngby, 1992.

[19] Eriksson J, Lindstrom P. A parallel interval method implementation for global optimization using dynamic load balancing. Reliable Computing, 1995, 1(1):77-92.

[20] Benyoub A, Daoudi E M. Parallelization of the continuous global optimization problem with inequality constraints by using interval arithmetic. Proceedings of the 9th International Conference on HighPerformance Computing and Networking, 2001, pp.595-602. 
[21] Ibraev S. A new parallel method for verified global optimization $\mathrm{PhD}$ Thesis. University of Wuppertal, Germany, 2001.

[22] Gau C Y, Stadtherr M A. Parallel intervalNewton using message passing: dynamic load balancing strategies. Supercomputing '01: Proceedings of the 2001 ACM/IEEE Conference on Supercomputing (CDROM), Denver, Colorado, 2001, pp.23-23.

[23] Berner S. Parallel methods for verified global optimization: practice and theory. Journal of Global Optimization, 1996, 9:122 .

[24] Message Passing Interface Forum. MPI: The message passing interface standard. 1994.

[25] Wu Y, Kumar A. Interval subdivision strategies for constrained optimization. Proceedings of the International Conference of Numerical Analysis and Applied Mathematics 2005, 2005.

[26] Ratschek H, Rokne J. New Computer Methods for Global Optimization. New York / Chichester / Brisbane / Toronto: Ellis Horwood Limited, 1988.
[27] Floudas C A, Pardalos P M, Adjiman C S, Esposito W R, Gümüs Z H, Harding S T, Klepeis J L, Meyer C A, Schweiger C A. Handbook of Test Problems in Local and Global Optimization. Dordrecht / Boston / London: Kluwer Academic Publishers, 1999.

[28] Michalewicz Z. Genetic Algorithms + Data Structures $=$ Evolution Programs. Berlin / Heidelberg / New York: Springer, 1996.

[29] Törn A, Žilinskas A. Global Optimization, Vol. 350 of Lecture Notes in Computer Science. Berlin: Springer-Verlag, 1989.

[30] Mäkelä M M, Neittaanmäki P. Nonsmooth Optimization. Singapore / New Jersey / London / Hong Kong: World Scientific, 1992.

[31] CUTEr. CUTEr: A constrained and unconstrained testing environment, revisited. http://cuter.rl.ac.uk/cutewww/problems.html.

[32] Knüppel O. PROFILE/BIAS - a fast interval library. Computing, 1994, 53:277287. 